\title{
FROM EDELWEISS-I TO EDELWEISS-II
}

\author{
V. SANGLARD \\ FOR THE EDELWEISS COLLABORATION \\ Institut de Physique Nucléaire de Lyon, \\ Université Claude Bernard Lyon 1, \\ 43, Bd du 11 Novembre 1918, \\ 69622 Villeurbanne Cedex, FRANCE \\ E-mail: sanglard@ipnl.in2p3.fr
}

\begin{abstract}
The EDELWEISS experiment is a Direct Dark Matter Search using $320 \mathrm{~g}$ heat-andionization Ge cryogenic detectors. The final results obtained by the EDELWEISSI stage corresponding to a total of $62 \mathrm{~kg}$.day are presented. The status of EDELWEISS-II, involving in a first phase $\sim 10 \mathrm{~kg}$ of detectors and aiming to gain two orders of magnitude in sensitivity, is also described.
\end{abstract}

\section{Introduction}

The EDELWEISS experiment is dedicated to the search for non-baryonic cold dark matter in the form of WIMPs (Weakly Interactive Massive Particles). The direct detection principle consists in the measurement of the energy released by nuclear recoils produced in an ordinary matter target by elastic collisions of WIMPs from the galactic halo.

The EDELWEISS detectors are cryogenic Ge bolometers with simultaneous measurement of phonon and ionization signals. The comparison of the two signals provides an excellent event-by-event discrimination between nuclear recoils (induced by WIMP or neutron scattering) and electronic recoils (induced by $\beta$ or $\gamma$-radioactivity).

The experiment is located in the Modane Underground Laboratory in the tunnel connecting France and Italy under $\sim 1800 \mathrm{~m}$ of rock ( $\sim 800 \mathrm{mwe})$. In the laboratory, the resulting muon flux is $4 \mu / \mathrm{m}^{2} / \mathrm{d}$ and the fast neutron flux has been measured ${ }^{1}$ to be $\sim 1.6 \times 10^{-6} \mathrm{~cm}^{2} / \mathrm{s}$.

During three years, $62 \mathrm{~kg}$.day of data have been accumulated with five $320 \mathrm{~g}$ Ge detectors. 


\section{Experimental set-up}

Between 2002 and 2003, three 320 g Ge detectors were operated simultaneously in a dilution cryostat with a regulated temperature of $17 \pm 0.01 \mathrm{mK}$. A passive shielding made of paraffin $(30 \mathrm{~cm})$, lead $(15 \mathrm{~cm})$ and copper $(10 \mathrm{~cm})$ surrounded the experiment ${ }^{2,3}$.

The detectors ${ }^{4}$ are made of a cylindrical Ge crystal with $\mathrm{Al}$ electrodes to collect ionization signals and a NTD heat sensor glued onto one electrode to collect the phonon signal. The top electrode is segmented in a central electrode and an annular guard ring and defines a fiducial volume corresponding to $57 \pm 2 \%$ of the total volume ${ }^{5}$. On four of the five detectors used in EDELWEISS-I an amorphous layer (either of Ge or $\mathrm{Si}$ ) was deposited under the electrodes to improve charge collection of near surface events ${ }^{6}$.

The resolutions and energy thresholds of the detectors are summarized in Ref. ${ }^{5}$. Detectors with an amorphous layer show a $99.99 \%$ gamma rejection at a recoil energy of $100 \mathrm{keV}$ and a $99.9 \%$ gamma rejection at a recoil energy of $15 \mathrm{keV}$.

\section{Edelweiss-I results}

During the EDELWEISS-I stage (2000-2003), four physics runs have been performed with five detectors. In the three first runs, the trigger was the fast ionization signal. For the last run, the trigger was the phonon signal. Thanks to a better resolution and the absence of quenching factor on the phonon signal, the phonon trigger improves the efficiency at low energy for nuclear recoils. In this last configuration, a $100 \%$ efficiency has been reached at $15 \mathrm{keV}$ on the three detectors. With the ionization trigger a $\sim 100 \%$ efficiency was reached at $20 \mathrm{keV}$ or $30 \mathrm{keV}$, depending on the detector. The low-background physics data recorded in the phonon trigger configuration are shown in Fig. 1.

Considering the complete $62 \mathrm{~kg}$.day data set, 60 events compatible with nuclear recoils have been recorded above a recoil energy of $10 \mathrm{keV}$. The corresponding energy spectrum is shown in Fig. 2, compared with simulations of theoretical spectrum for different WIMP masses, taking into account the recoil energy dependence efficiency ${ }^{\mathrm{a}}$ of all experimental configurations.

\footnotetext{
aThe efficiency calculation takes into account thresholds, resolutions, the $90 \%$ efficiency $(1.65 \sigma)$ for the nuclear recoil band and the $99.9 \%$ rejection $(3.29 \sigma)$ of the electronic recoils.
} 


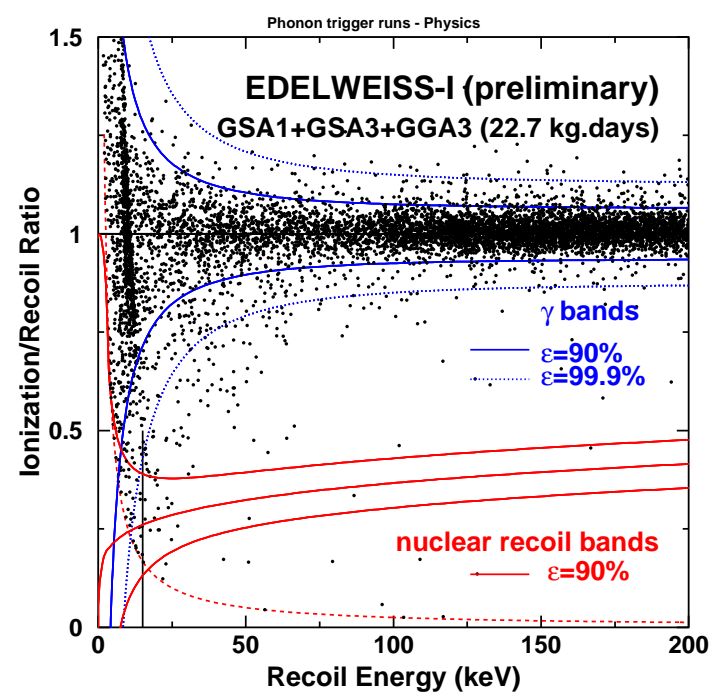

Figure 1. $\frac{E_{I}}{E_{R}}$ versus $E_{R}$ (fiducial volume) for physics runs with the phonon trigger configuration.

The overall shape of the experimental spectrum is incompatible with WIMP masses above $20 \mathrm{GeV} / \mathrm{c}^{2}$.

Setting our analysis threshold to $20 \mathrm{keV}$, above which the experimental efficiency is greater than $75 \%, 23$ events compatible with nuclear recoils have been observed. Considering all these events as possible WIMP interactions and taking into account the efficiency versus recoil energy function of each run ${ }^{\mathrm{b}}$, a conservative upper limit on the WIMP-nucleon cross-section as a function of the WIMP mass has been derived with the Optimum Interval Method ${ }^{7}$. This method allows to compute an exclusion limit in the presence of an unknown background. Fig. 3 shows the EDELWEISS-I spin independent exclusion limit, assuming a standard spherical and isothermal galactic WIMP halo with a local density of $0.3 \mathrm{GeV} / \mathrm{c}^{2} / \mathrm{cm}^{3}$, a rms velocity of $270 \mathrm{~km} / \mathrm{s}$, an escape velocity of $650 \mathrm{~km} / \mathrm{s}$ and a relative Earth-halo velocity of $230 \mathrm{~km} / \mathrm{s}$. Limits from other running experiments are also shown

${ }^{\mathrm{b}}$ For example, for the complete data set of Edelweiss-I a $50 \%$ efficiency is reached for a recoil energy of $15 \mathrm{keV}$. 


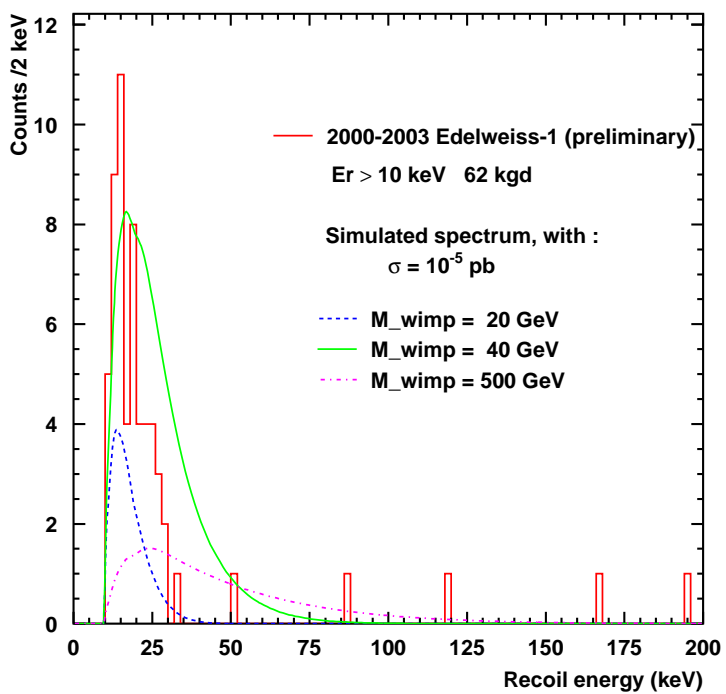

Figure 2. Energy spectrum for the EDELWEISS-I data, for $E_{R}>10 \mathrm{keV}$, compared to simulated theoretical WIMP spectrum for $\mathrm{M}_{W I M P}=20,40,500 \mathrm{GeV} / \mathrm{c}^{2}$.

on Fig. 3. With no background subtraction and an extended exposure, the new limit is consistent with the previous published one ${ }^{8}$.

Although the EDELWEISS-I limit of Fig. 3 is derived assuming all events as possible WIMP candidates, the experimental data reveal some clues as to the nature of possible backgrounds. For example, a two detector coincidence between nuclear recoils has been recorded. This event is very likely a neutron-neutron coincidence, indicating that a certain fraction of events in Fig. 2 could be due to single hits by neutrons. Miscollected charge events, as indicated by the few events lying between electronic and nuclear recoil bands in Fig. 1, are another possible source of background, because they can simulate nuclear recoils. But with the present statistics, limited largely by the number of detectors (the EDELWEISS-I cryostat could not receive more than $3 \times 320$ g detectors), it is not possible to conclude any further.

\section{Lessons for EDELWEISS-II}

The second phase of the experiment will be EDELWEISS-II, with an expected sensitivity of $0.002 \mathrm{evt} / \mathrm{kg} / \mathrm{d}$. Specific improvements are aimed at 


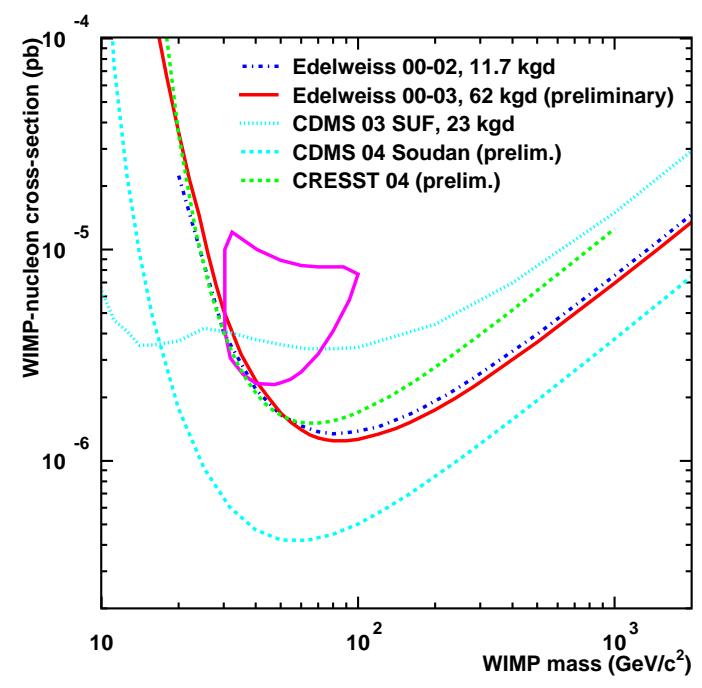

Figure 3. Preliminary spin independent exclusion limit for EDELWEISS-I without background subtraction compared to CDMS limit, when the experiment was operated at Stanford ${ }^{9}$ and in the Soudan mine ${ }^{10}$, and the CRESST limit ${ }^{11}$. The closed contour is the allowed region at $3 \sigma$ C.L. from DAMA NaI1- 4 annual modulation data ${ }^{12}$.

reducing the possible background sources, that may have limited the sensitivity of EDELWEISS-I. In addition, the detector number will be increased up to 28 to achieve a Ge mass of $\sim 10 \mathrm{~kg}$ in a first stage.

\subsection{Neutrons}

The low energy neutron background, due to the radioactive surrounding rock, is attenuated by more than three orders of magnitude thanks to a $50 \mathrm{~cm}$ polyethylene shielding. In addition, a muon veto ${ }^{14}$ surrounding the experiment will tag muons interacting in the lead shielding. The increased number of detectors will improve the possibility of detecting multiple interactions of neutrons.

\subsection{Surface events}

Surface events, namely interactions near electrodes, show a deficit of the charge collection. One of the R\&D goals in EDELWEISS is the event-by- 
event identification of these miscollected events and their active rejection. A new generation of detectors has been developped with $\mathrm{NbSi}$ thin film sensors (instead of the NTD heat sensors for present detectors). They consist in a Ge crystal with two NbSi sensors acting also as electrodes for charge collection. These thin film sensors are sensitive to the athermal component of the phonon signal, acting as near-surface interaction tag ${ }^{13}$. Several tests have been made in the EDELWEISS-I setup with three $200 \mathrm{~g}$ Ge detectors showing a reduction by a factor 10 of the surface event rate while retaining a $50 \%$ efficiency. Seven $400 \mathrm{~g}$ Ge detectors are being prepared in a first stage for EDELWEISS-II.

Furthermore, improved radiopurity and clean room conditions are expected to reduce the contaminations and the rate of surface electrons.

\section{Conclusion}

EDELWEISS-I experiment has reached its limit sensitivity near $10^{-6} \mathrm{pb}$, allowing the exclusion of some optimistic SUSY models. The goal for the future with EDELWEISS-II is to reach more favored models close to $10^{-8} \mathrm{pb}$. The EDELWEISS-I experiment was stopped in March 2004 to allow the installation of the second phase EDELWEISS-II. The first runs will be performed with $21 \times 320 \mathrm{~g}$ Ge detectors with NTD heat sensor and $7 \times 400 \mathrm{~g}$ Ge detectors with NbSi thin film sensor. Data taking in the new setup is scheduled for end-2005.

\section{References}

1. G. Chardin and G. Gerbier, in Proceedings of th 4 th International Workshop on Identification of Dark Matter (IDM2002), eds N.J. Spooner

2. A. Benoit et al., Phys. Lett. B, 513, 15 (2001)

3. Ph. di Stefano et al., Astropart. Phys., 14, 329 (2001)

A. de Bellefon et al., Astropart. Phys., 6, 35 (1996)

4. X.F. Navick et al., Nucl. Inst. Meth. A, 444, 361 (2000)

5. O. Martineau et al., Nucl. Inst. Meth. A, 530, 426 (2004)

6. T. Shutt et al. , Nucl. Inst. Meth. A, 444, 340 (2000)

7. S. Yellin, Phys. Rev. D, 66, 032005 (2002)

8. A. Benoit et al., Phys. Lett. B 545, 43 (2002).

9. D.S. Akerib et al., Phys. Rev. D, 68, 082002 (2003)

10. D.S. Akerib et al., astro-ph/0405033, submitted to Phys. Rev. Lett

11. G. Angloher et al., astro-ph/0408006, submitted to Astropart. Phys.

12. R. Bernabei et al, Phys. Lett. B, 480, 23 (2000)

13. S. Marnieros et al, Nucl. Inst. Meth. A, 520, 185 (2004)

14. L. Chabert, Ph. D. Thesis (unplubished), Université Claude Bernard Lyon 1, France (July 7th, 2004), available on http://tel.ccsd.cnrs.fr 